\title{
Transient Pseudohypoaldosteronism Secondary to Group B Streptococcus Pyelonephritis
}

\author{
Atsuo Morisaki ${ }^{1}$, Yuki Naruse ${ }^{1}$, Yui Shibata ${ }^{1}$, Masato Mori ${ }^{1}$, Ryugo Hiramoto ${ }^{1}$ \\ 1. Pediatrics, Children Center, Matsudo City General Hospital, Matsudo, JPN
}

Corresponding author: Yuki Naruse, cellist670573yuuki@yahoo.co.jp

\begin{abstract}
Secondary pseudohypoaldosteronism is a condition characterized by aldosterone resistance in renal tubules. It is highly associated with urinary tract infection and urinary tract malformations. Only a few cases of pseudohypoaldosteronism secondary to group B Streptococcus pyelonephritis have been reported to date. A four-month-old boy developed poor sucking and weight loss, and his laboratory test results revealed hyponatremia, hyperkalemia, renal dysfunction, high anion gap metabolic acidosis, pyuria, and hydronephrosis. Laboratory tests including urinalysis confirmed the diagnosis of pseudohypoaldosteronism secondary to group B Streptococcus. He was treated with intravenous normal saline and antimicrobial therapy. Electrolyte disorders were addressed and he was discharged on the 10th day of hospitalization without any sequelae. Voiding cystourethrography performed after discharge showed bilateral grade 5 vesicoureteral reflux and intrarenal reflux in the right kidney. Transient pseudohypoaldosteronism is an important consideration in the differential diagnosis in infants with hyponatremia and hyperkalemia. A thorough evaluation for urinary tract malformations should be performed, including early abdominal ultrasonography and systemic management.
\end{abstract}

Review began 05/07/2021 Review ended 05/14/2021 Published 05/17/2021

\section{(๑) Copyright 2021}

Morisaki et al. This is an open access article distributed under the terms of the Creative Commons Attribution License CC-BY 4.0., which permits unrestricted use, distribution, and reproduction in any medium, provided the original author and source are credited.
Categories: Endocrinology/Diabetes/Metabolism, Pediatrics, Nephrology

Keywords: group b streptococcus, pseudohypoaldosteronism, hyperkalemia, urinary tract malformations, urinary tract infections

\section{Introduction}

Type 1 pseudohypoaldosteronism is a condition characterized by hyponatremia, hyperkalemia, and metabolic acidosis. It is associated with aldosterone resistance in renal tubules. The syndrome is subdivided into a genetic form and secondary form. Secondary pseudohypoaldosteronism is known to be associated with urinary tract malformations and urinary tract infections [1,2]. Escherichia coli is the most common pathogen causing urinary tract infections in patients with secondary pseudohypoaldosteronism [2]. Only a few cases of pseudohypoaldosteronism secondary to group B Streptococcus (GBS) infection have been reported to date. We report a case of a four-month-old boy who developed poor sucking and weight loss and was diagnosed with GBS pyelonephritis and a urinary tract malformation.

\section{Case Presentation}

A previously healthy four-month-old male infant was referred and subsequently admitted to our hospital with poor sucking that started two days prior to presentation. He was born healthy at 40 weeks and 0 days of gestation by normal vaginal delivery. There was no history of any maternal infection. His birth weight was $3454 \mathrm{~g}$, which was appropriate for his gestational age. He had been exclusively breastfed and was well with a weight of $6300 \mathrm{~g}$ at his four-month supervision one week prior to admission. On admission, he was $63.6 \mathrm{~cm}$ $(-0.26 \mathrm{SD})$ in length and $5670 \mathrm{~g}$ in weight, which was $10 \%$ less than his weight from the previous examination. His vital signs were stable except for mild hypotension (blood pressure, $69 / 48 \mathrm{mmHg}$; heart rate, $122 \mathrm{bpm}$; respiratory rate, $34 / \mathrm{min}$; body temperature. $36.7^{\circ} \mathrm{C}$; and $\mathrm{SpO}_{2}$ at room air, $100 \%$ ). On physical examination, facial pallor, sunken eyes and fontanel, dry mucous membranes, mild delay in skin elasticity, delayed capillary refill ( $2 \mathrm{~s})$, reticular cyanosis, and coldness of extremities were noted. Cardiac and respiratory examination findings were unremarkable. There were no external genital malformations, and external genital or general skin pigmentation.

Blood test results (Table 1) showed hyponatremia (serum Na+, $114 \mathrm{mEq} / \mathrm{L}$ ), hyperkalemia (serum K+, 9.3 $\mathrm{mEq} / \mathrm{L}$ ), renal dysfunction, and high anion gap metabolic acidosis. There was no hypoglycemia. Inflammatory markers were not elevated. Urinalysis revealed pyuria, hematuria, and proteinuria. Chest and abdominal $\mathrm{x}$-rays revealed no abnormalities. Ultrasonography showed no abnormalities in the head or heart but revealed left hydronephrosis (grade unknown). Electrocardiography showed peaked T waves but no QT prolongation (QTc, $437 \mathrm{~ms})$. 


\section{Cureus}

\begin{tabular}{|c|c|c|c|c|c|c|c|}
\hline Test & Value & & Normal Range & Test & Value & & Normal Range \\
\hline \multicolumn{3}{|c|}{ Complete Blood Count } & & \multicolumn{2}{|c|}{ Biochemistry } & & \\
\hline WBC & 20,000 & $/ \mu \mathrm{L}$ & $(4600-18,900)$ & AST & 88 & U/L & $(22-66)$ \\
\hline $\mathrm{N}$ & 41 & $\%$ & $(20-50)$ & ALT & 79 & $U / L$ & $(13-56)$ \\
\hline L & 52 & $\%$ & (44-74) & LDH & 249 & $U / L$ & (205-418) \\
\hline RBC & 507 & $10^{4} / \mu \mathrm{L}$ & $(340-500)$ & BUN & 66.4 & $\mathrm{mg} / \mathrm{dL}$ & $(2.2-14.1)$ \\
\hline $\mathrm{Hb}$ & 12.9 & $\mathrm{~g} / \mathrm{dL}$ & $(9.5-13.7)$ & Cre & 0.96 & $\mathrm{mg} / \mathrm{dL}$ & $(0.12-0.27)$ \\
\hline Hct & 38.8 & $\%$ & $(28.5-41.1)$ & $\mathrm{Na}$ & 114 & $\mathrm{mEq} / \mathrm{L}$ & (135-143) \\
\hline \multirow[t]{2}{*}{ PIt } & 77.3 & $10^{3} / \mu \mathrm{L}$ & $(25.0-82.0)$ & $\mathrm{Cl}$ & 88 & $\mathrm{mEq} / \mathrm{L}$ & $(101-111)$ \\
\hline & & & & $\mathrm{K}$ & 9.3 & $\mathrm{mEq} / \mathrm{L}$ & $(4.1-5.6)$ \\
\hline \multicolumn{2}{|c|}{ Blood Gas (Vein) } & & & CRP & 0.69 & $\mathrm{mg} / \mathrm{dL}$ & $(<0.30)$ \\
\hline $\mathrm{pH}$ & 7.178 & & (7.36-7.44) & & & & \\
\hline $\mathrm{pCO}_{2}$ & 25.6 & $\mathrm{mmHg}$ & $(36-44)$ & \multicolumn{2}{|c|}{ Endocrine Examination } & & \\
\hline $\mathrm{HCO}_{3}{ }^{-}$ & 9.3 & $\mathrm{mmol} / \mathrm{L}$ & $(22-26)$ & ACTH & 6.6 & $\mathrm{pg} / \mathrm{mL}$ & $(11.6-35.0)$ \\
\hline BE & -17.4 & $\mathrm{mmol} / \mathrm{L}$ & $(-2 \sim+3)$ & Cortisol & 47.3 & $\mu \mathrm{g} / \mathrm{dL}$ & $(3.0-23.0)$ \\
\hline Lac & 4.6 & $\mathrm{mmol} / \mathrm{L}$ & $(<2.0)$ & PRA & $>20$ & $\mathrm{ng} / \mathrm{mL} / \mathrm{h}$ & $(0.3-2.9)$ \\
\hline Glu & 160 & $\mathrm{mg} / \mathrm{dL}$ & $(50-109)$ & PAC & 42,700 & $\mathrm{pg} / \mathrm{mL}$ & $(35.7-240)$ \\
\hline
\end{tabular}

\section{TABLE 1: Blood Test Results}

WBC: white blood cells; N: neutrophil; L: lymphocyte; RBC: red blood cells; Hb: hemoglobin; Hct: hematocrit; PIt: platelets; BE: base excess; Lac: lactate; Glu: glucose; AST: aspartate transaminase; ALT: alanine aminotransferase; LDH: lactate dehydrogenase; BUN: blood urea nitrogen; Cre: creatinine; CRP: C-reactive protein; ACTH: adrenocorticotropin; PRA: plasma renin activity; PAC: plasma aldosterone concentration

Aldosterone deficiency was suspected owing to the presence of hyponatremia, hyperkalemia, metabolic acidosis, and dehydration. His plasma aldosterone concentration was elevated, which ruled out aldosterone deficiency. Adrenal insufficiency was also considered in the differential diagnosis but was less likely because of the absence of hypoglycemia. The patient was diagnosed with secondary pseudohypoaldosteronism owing to his high plasma renin activity and high serum aldosterone levels in his admission samples.

Adrenocorticotropin (ACTH) assay was within normal ranges and serum cortisol assay was elevated, which excluded adrenal insufficiency. Cortisol replacement was not performed. Electrolyte abnormalities were corrected with rapid infusion of normal saline, glucose-insulin therapy, intravenous calcium gluconate hydrate, sodium bicarbonate, and oral cation exchange resin. Electrolyte abnormalities improved within $4 \mathrm{~h}$ of admission.

The patient did not have any episodes of fever; however, he was treated with cefotaxime $200 \mathrm{mg} / \mathrm{kg} / \mathrm{day}$ and ampicillin (ABPC) $200 \mathrm{mg} / \mathrm{kg} /$ day because of leukocytosis and pyuria. Although abdominal ultrasonography on admission showed left hydronephrosis, no hydronephrosis was found in the repeat examination on the second day of admission. On the fifth day of hospitalization, GBS was detected in the urine culture at a concentration of $10^{8} / \mathrm{mL}$, and the antimicrobial agent was changed to ABPC monotherapy. Blood culture was sterile. He was discharged on the 10th day of hospitalization.

After discharge from the hospital, voiding cystourethrogram (Figure 1) was performed, which revealed bilateral grade 5 vesicoureteral reflux and intrarenal reflux in the right kidney. On the 28th day, plasma renin activity and serum aldosterone levels improved to $4.0 \mathrm{ng} / \mathrm{mL} / \mathrm{h}$ and $679 \mathrm{pg} / \mathrm{mL}$, respectively. Two months after discharge from the hospital, ureteroneocystostomy was performed. The patient progressed without subsequent recurrence of urinary tract infection. At the age of two years and five months, he was $92.0 \mathrm{~cm}$ $(+0.96 \mathrm{SD})$ in height and $12.7 \mathrm{~kg}(+0.94 \mathrm{SD})$ in weight, with good growth and normal neurodevelopment. 


\section{Cureus}

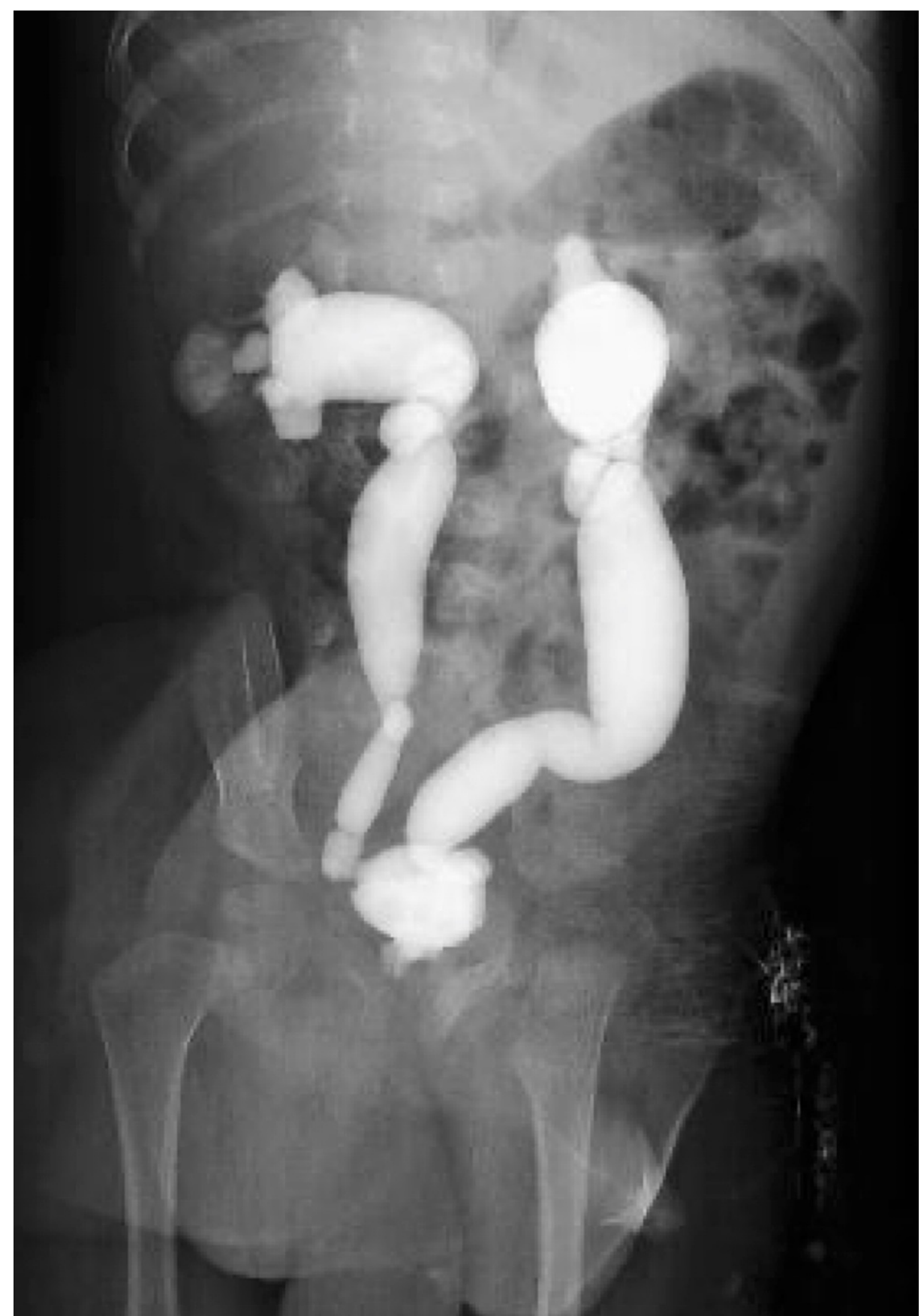

\section{FIGURE 1: Voiding Cystourethrogram}

Voiding cystourethrogram revealed bilateral grade 5 vesicoureteral reflux and intrarenal reflux in the right kidney

\section{Discussion}

Since Rodríguez-Soriano et al. reported the first pediatric case of secondary pseudohypoaldosteronism in 1983 [3], more than 100 cases have been reported. Secondary pseudohypoaldosteronism mainly occurs in infants younger than three months and is highly associated with male gender, urinary tract infection, and urinary tract malformations [1]. In Delforge's review of secondary pseudohypoaldosteronism caused by urinary tract malformations [4], the condition was more frequently associated with male gender (81.9\%) and urinary tract infection (90.5\%). Among urinary tract malformations, vesicoureteral reflux (47.4\%) was more common.

GBS is an unusual urinary tract pathogen and is highly associated with urinary tract malformation in children [5,6]. Only a few cases of GBS-related pseudohypoaldosteronism have been reported to date [7-9]. GBS urinary tract infection in an infant may result from hematogenous seeding of bacteria [10]; however, the 
blood culture was sterile in our case. Therefore, our patient was diagnosed with an ascending urinary tract infection. According to the age of onset, GBS infections are classified as early-onset, late-onset, or late-lateonset disease (LLOD). Our patient's infection was classified as LLOD because the onset started after the age of 89 days. LLOD accounts for $10 \%$ of all infantile GBS infections and typically occurs in infants who were delivered at less than 35 weeks of gestation [11]; however, our patient was delivered vaginally at full term and had no history of maternal infection.

Secondary pseudohypoaldosteronism mainly causes nonspecific symptoms, such as poor feeding, poor weight gain, vomiting, failure to thrive, and dehydration [1]. Some cases have reported convulsive seizures with sequelae, fatal arrhythmias, and cardiac arrest [12-14]. The symptoms resolve with intravenous fluids, electrolyte correction, and antibiotic therapy, and the prognosis is good. Therefore, early diagnosis is required to prevent serious complications and sequelae.

Congenital adrenocortical hyperplasia and congenital adrenal hypoplasia are considered in the differential diagnoses of salt-losing nephropathy accompanied by hyperkalemia [15]. Our patient had no external genital malformations, external genital or general skin pigmentation, or hypoglycemia; therefore, these conditions were unlikely. The patient had pyuria on urinalysis and hydronephrosis on ultrasonography and was treated according to the first diagnosis of secondary pseudohypoaldosteronism. After electrolyte correction on admission, the electrolytes remained stable. The patient was diagnosed with secondary pseudohypoaldosteronism based on the results of plasma renin, serum aldosterone, ACTH, and serum cortisol levels obtained at the time of admission. ACTH levels were slightly low but might have been caused by the time taken from sample collection to plasma separation. Adrenal insufficiency-related syndrome could have been included in the differential diagnosis based on the initial general condition of the patient. A therapeutic strategy of concomitant glucocorticoid supplementation could have been considered since cases of initial treatment with glucocorticoids have been reported $[1,13]$.

The pathophysiology of secondary pseudohypoaldosteronism remains unclear. Approximately $80 \%$ of patients with transient pseudohypoaldosteronism are less than seven months old [16]; therefore, it is speculated that tubular immaturity may contribute to the pathogenesis of the disease because this would require high aldosterone levels to maintain electrolyte balance [4]. Infection and urinary tract obstruction are considered to cause increased aldosterone resistance in renal tubules, both directly through the impairment of cellular response mechanisms and indirectly through changes in renal hormone levels [17]. The onset of secondary pseudohypoaldosteronism occurred at four months of age in our case, suggesting that this condition should be considered in the differential diagnosis of hyponatremia and hyperkalemia in early infancy.

\section{Conclusions}

A four-month-old boy who developed poor sucking and weight loss was diagnosed with pseudohypoaldosteronism secondary to GBS pyelonephritis. Pseudohypoaldosteronism is important in the differential diagnosis in early infants with hyponatremia and hyperkalemia. A thorough evaluation for urinary tract malformations should be performed, including abdominal ultrasonography at an early stage, alongside systemic management.

\section{Additional Information}

\section{Disclosures}

Human subjects: Consent was obtained or waived by all participants in this study. Conflicts of interest: In compliance with the ICMJE uniform disclosure form, all authors declare the following: Payment/services info: All authors have declared that no financial support was received from any organization for the submitted work. Financial relationships: All authors have declared that they have no financial relationships at present or within the previous three years with any organizations that might have an interest in the submitted work. Other relationships: All authors have declared that there are no other relationships or activities that could appear to have influenced the submitted work.

\section{Acknowledgements}

Thank Editage (https://www.editage.jp/) for the English language review.

\section{References}

1. Bogdanović R, Stajić N, Putnik J, Paripović A: Transient type 1 pseudo-hypoaldosteronism: report on an eight-patient series and literature review. Pediatr Nephrol. 2009, 24:2167-75. 10.1007/s00467-009-1285-8

2. Melzi ML, Guez S, Sersale G, et al.: Acute pyelonephritis as a cause of hyponatremia/hyperkalemia in young infants with urinary tract malformations. Pediatr Infect Dis J. 1995, 14:56-9. 10.1097/00006454-19950100000012

3. Rodríguez-Soriano J, Vallo A, Oliveros R, Castillo G: Transient pseudohypoaldosteronism secondary to obstructive uropathy in infancy. J Pediatr. 1983, 103:375-80. 10.1016/s0022-3476(83)80406-5

4. Delforge X, Kongolo G, Cauliez A, Braun K, Haraux E, Buisson P: Transient pseudohypoaldosteronism: a 
potentially severe condition affecting infants with urinary tract malformation. J Pediatr Urol. 2019, 15:265.e1-7. 10.1016/j.jpurol.2019.03.002

5. Schlager TA: Urinary tract infections in infants and children. Microbiol Spectr. 2016,

4:10.1128/microbiolspec.UTI-0022-2016

6. Murphy AV, Shaw R, McAllister TA: Significance of group B streptococci (Strep agalactiae) in children's urinary tract infection. Lancet. 1987, 1:98-9. 10.1016/s0140-6736(87)91929-5

7. Kashimada K, Omori T, Takizawa F, Mizutani S: Two cases of transient pseudohypoaldosteronism due to group B streptococcus pyelonephritis. Pediatr Nephrol. 2008, 23:1569-70. 10.1007/s00467-008-0843-9

8. Xu M, Di Blasi C, Dickerson J, Jack R, Rutledge JC: A 5-week-old boy with failure to thrive, marked hyperkalemia, and hyponatremia. Clin Chem. 2016, 62:1439-43. 10.1373/clinchem.2015.252320

9. Nandagopal R, Vaidyanathan P, Kaplowitz P: Transient pseudohypoaldosteronism due to urinary tract infection in infancy: a report of 4 cases. Int J Pediatr Endocrinol. 2009, 2009:195728. 10.1155/2009/195728

10. Yagupsky P, Menegus MA, Powell KR: The changing spectrum of group B streptococcal disease in infants: an eleven-year experience in a tertiary care hospital. Pediatr Infect Dis J. 1991, 10:801-8. 10.1097/00006454199111000-00002

11. Edwards MS, Nizet V, Baker CJ: Group B streptococcal infections. Remington and Klein's Infectious Diseases of the Fetus and Newborn Infant, 8th ed. Wilson CB, Nizet V, Maldonado YA, Remington JS, Klein JO (ed): Saunders Elsevier, Philadelphia, PA; 2015. 411-456.

12. Belot A, Ranchin B, Fichtner C, et al.: Pseudohypoaldosteronisms, report on a 10-patient series. Nephrol Dial Transplant. 2008, 23:1636-41. 10.1093/ndt/gfm862

13. Thies KC, Boos K, Müller-Deile K, Ohrdorf W, Beushausen T, Townsend P: Ventricular flutter in a neonatesevere electrolyte imbalance caused by urinary tract infection in the presence of urinary tract malformation. J Emerg Med. 2000, 18:47-50. 10.1016/s0736-4679(99)00161-4

14. Kibe T, Sobajima T, Yoshimura A, Uno Y, Wada N, Ueta I: Secondary pseudohypoaldosteronism causing cardiopulmonary arrest and cholelithiasis. Pediatr Int. 2014, 56:270-2. 10.1111/ped.12267

15. Amin N, Alvi NS, Barth JH, et al.: Pseudohypoaldosteronism type 1: clinical features and management in infancy. Endocrinol Diabetes Metab Case Rep. 2013, 2013:130010. 10.1530/EDM-13-0010

16. Watanabe T: Reversible secondary pseudohypoaldosteronism. Pediatr Nephrol. 2003, 18:486. 10.1007/s00467-003-1104-6

17. Giapros VI, Tsatsoulis AA, Drougia EA, Kollios KD, Siomou EC, Andronikou SK: Rare causes of acute hyperkalemia in the 1st week of life. Three case reports. Pediatr Nephrol. 2004, 19:1046-9. 10.1007/s00467004-1500-6 